\title{
OPTICAL PROPERTIES OF B2 RADIO GALAXIES
}

\author{
J.I. GONZÁLEZ-SERRANO AND R. CARBALLO \\ Instituto de Física de Cantabria, Santander, Spain
}

\begin{abstract}
We present a large program of CCD imaging of low-luminosity radio galaxies selected from the $B 2$ survey of radio sources. We aim to study their optical properties: brightness profiles, isophotal distortions, morphological peculiarities. As the sample contains jet and no-jet sources, this investigation will allow us to compare both populations of low-luminosity radio galaxies. In particular, we will study the local environment and test whether the galaxy density around radio sources plays an important role on the presence of jets or not. The analysis of morphological distortions on the host galaxies will provide us with detailed information on past or recent galaxy encounters.
\end{abstract}

\section{The sample}

The sample of low-luminosity radio galaxies is described in Parma et al. (1987) (and references therein). It contains $\sim 110$ sources identified with galaxies in the POSS plates and mapped using the VLA at $1.4 \mathrm{GHz}$. About $45 \%$ of the sources present a radio jet at the kpc scale. The radio power of these sources at $1.4 \mathrm{GHz}$ is less than $10^{25} \mathrm{~W} \mathrm{~Hz}^{-1}$ and their redshifts are less than $\sim 0.15$.

\section{Previous results}

We continue a study on the optical properties of low-luminosity radio galaxies based on broad-band imaging of this complete sample of $\mathrm{B} 2$ radio sources. We have finished the study of those sources containing radio jets at the kpc scale on VLA maps. In González-Serrano et al. (1993) we conclude that low-luminosity radio galaxies with jets inhabit regions of higher galaxy density than powerful radio galaxies. We have found that a high fraction of 
this kind of radio sources show indications of past/recent mergers/collisions with another (elliptical) galaxy.

Here we present CCD imaging of this sample of radio sources. The sources are similar to the radio jet sample but they have no jets at the kpc scale on similar VLA maps. In that way we can compare the properties of two populations selected from exactly the same sample.

\section{Present results}

We are starting now to analyse the non-jet sample in the same way as we did with the jet sample. This will allow to investigate how important is the local galaxy density in the triggering of large scale radio jets, and if merging processes have some role on the presence of jets.

The observations were carried out at the JKT $1 \mathrm{~m}$ telescope of the Observatorio del Roque de los Muchachos on the island of La Palma. We used a TEK detector and we took 1800 sec integrations in filter V. Pixel scale and field of view are $0.34 \mathrm{arcsec} / \mathrm{pixel}$ and $5.8 \times 5.8 \mathrm{arcmin}$ respectively. The observations were done on January and May 1995. Preliminary reduction has been done in the standard way. Isophotal analysis and local galaxy density measurements are in progress.

From visual inspection of the contour maps of the galaxies we notice that there is a fraction of spiral and irregular galaxies which are absent in the jet subsample. We can also see a fraction of distorted galaxies although it seems to be a fraction not as high as it appears to be in the radio jet sample. We also notice that there are few galaxies with companions, while in the jet subsample this is a common feature. In summary, a preliminary inspection of the optical counterparts of the no-jet sources seems to indicate that the environments of both populations are different, being more dense in radio jet sources. Of course, this should be investigated in more detail using the same procedures that were used with the jet sample.

\section{References}

González-Serrano, J.I., Carballo, R., \& Pérez-Fournon, I. 1993, AJ, 105, 1710

Parma, P., Fanti, C., Fanti, R., Morganti, R., de Ruiter, H.R. 1987, A\&A, 181, 244 